\title{
GIULIO GHIRARDI, 2014, “Appunti e contrappunti”, Marcianum press, Venezia
}

Nell'edizione speciale del "Centro per lo Studio e la tutela dei Beni Culturali", non numerata e fuori serie della rivista "Arte | Documento - Rivista e Collezione di Storia e tutela dei Beni Culturali" (fondata nel 1987 per iniziativa della cattedra di Storia dell'Arte moderna dell'Università degli Studi di Udine, per poi essere curata, dal 1994, dall'omonima cattedra dell'Università Ca' Foscari di Venezia), è uscito dalle stampe un libro del dott. Giulio Ghirardi, apprezzato storico e critico d’arte, nonché scrittore italiano. Si tratta di un'antologia che raccoglie le più caratteristiche trattazioni su diversi intellettuali che Ghirardi aveva avuto modo di conoscere e con i quali aveva collaborato nel corso della sua vita.

Suddiviso in tre parti, il volume offre dodici testi, di carattere interdisciplinare, che pongono in luce il profondo interesse dell'autore per temi diversi, contraddistinti però da un comune elemento: Venezia, fonte di ispirazione letteraria onnipresente nelle opere di Ghirardi. A conclusione di quasi tutti i capitoli fanno bella mostra di sé alcune immagini che integrano ed arricchiscono armoniosamente la parte testuale.

Nella sua breve introduzione il prof. Giuseppe Maria Pilo, presidente del "Centro per lo Studio e la tutela dei Beni Culturali", fondatore e capo redattore della sunnominata rivista, pone l'accento sul fatto che il libro è un omaggio al dott. Ghirardi sotto forma di raccolta di saggi nei quali, scegliendo svariati stili di scrittura, lautore parla delle radici che lo legano all'arte. Pilo sottolinea che il libro ha per lui anche un significato sentimentale, visto che in qualità di direttore del Museo Civico di Pordenone aveva conosciuto Ghirardi nel lontano 1973. Già all'epoca notò in lui qualcosa di inedito e di insolito, vi riconobbe un personaggio che per allontanarsi dalle esperienze spiacevoli del mondo esterno si rifugiava in quello del racconto, della poesia e degli aforismi, sviluppando uno stile espressivo libero e indipendente, capace di abbracciare innumerevoli registri del pensiero umano. Nel farlo sceglieva liberamente quei temi che avevano attirato la sua attenzione dal 2000 in avanti, nel periodo di grande ispirazione dell'autore. 
Nel primo capitolo Ghirardi tratta il tema dei "tesori della memoria", ricordando soprattutto "Il flauto magico" ("Die Zauberflöte", 1791) di Mozart, la prima opera da lui ascoltata quand'era ancora bambino, nei primi anni Cinquanta. La definisce il "faro d'ispirazione per gli artisti del ventesimo secolo". Il passo inizia con un pensiero di Sant'Agostino: "attraverso la memoria il presente diventa passato e futuro: la memoria è un ponte senza il quale la storia di un uomo si sbriciola in un arcipelago di esperienze". Esistono la memoria indiretta, fatta passare attraverso il filtro dei ricordi di maturi testimoni, come pure la memoria diretta, che prende spunto da posizioni molto diverse tra loro. Il ricordo è sempre legato all'umore del momento ed a molte altre circostanze. Il "Flauto magico", al quale "ritorna nuovamente" dopo averlo assunto come tema già nel 1973, viene definito da Ghirardi come simbolo complesso posto nel contesto storicoideologico degli eventi dellepoca; egli prosegue poi descrivendo le celebrazioni del 1956 a Salisburgo in occasione del bicentenario della nascita di Mozart.

L'autore continua scrivendo del famoso commediografo italiano Carlo Goldoni (Venezia, 1707 - Parigi, 1793) ed analizzando alcune delle sue commedie nel contesto dell'epoca in cui l'autore visse ed operò: ne ricorda il duecentocinquantesimo anniversario della nascita, celebrato nella natia Venezia nel 1957 con varie manifestazioni e con numerose pubblicazioni. Apprezzandone l'ampiezza del pensiero creativo, l'autore pone in luce il fatto che il ricordo del grande commediografo non venne scalfito né dalla caduta della Repubblica di Venezia né dalle conquiste di Napoleone, e nemmeno dal dominio austriaco: lo si è sempre ricordato con "malinconica nostalgia", racconta Ghirardi.

Il terzo capitolo è dedicato a Benno Geiger (Rodaun, provincia di Vienna, 1882 - Venezia, 1965), poeta, traduttore, storico dell'arte e critico. Viennese di nascita, ma veneziano per il lungo periodo vissuto nella Città lagunare (lo stesso Ghirardi lo descrive come "personaggio di sangue viennese e modi lagunari"), Geiger era apprezzato dai suoi contemporanei come profondo conoscitore della lingua e della letteratura italiane. Notevole intellettuale, si distinse come oppositore del regime sorto dopo la Seconda guerra mondiale e contestò le nuove tendenze culturali del periodo. Nella sua opera "Memorie di un veneziano", della quale Ghirardi scrive, Geiger offre l'immagine della Venezia di un tempo, ormai scomparsa per sempre, descrivendo la propria vita ed i numerosi incontri con gli intellettuali suoi contemporanei.

Il pittore e scultore Umberto Boccioni (Reggio di Calabria, 1882 - Verona, 1916) e i suoi "Taccuini" del 1907-1908 sono al centro del capitolo successivo, nel quale l'autore discute delle opere di uno dei padri e dei principali teorici del Futurismo. Si ritiene che 
Boccioni fosse l'artista futurista di maggior talento e che la sua scomparsa precoce (a séguito di una caduta da cavallo sul fronte italiano settentrionale) segnò invero la fine del movimento.

Il nesso tra il compositore russo di origini ucraine Igor Stravinsky (Oranienbaum, 1882 - New York, 1971) e Venezia viene analizzato da Ghirardi nel quinto capitolo, molto più ampio dei restanti. Stravinsky è tuttora considerato da molti come il compositore che maggiormente influenzò la musica del XX secolo. Fu famoso anche come direttore d'orchestra e pianista, spesso esecutore delle prime delle sue stesse opere. Fu anche scrittore. Pur essendo morto in America, le sue spoglie riposano al cimitero veneziano di San Michele.

Il sesto capitolo ci offre i ricordi delle interviste che Giulio Ghirardi fece ad artisti del XX secolo che meritarono l'attenzione dell'autore grazie ad idee e ad opere di particolare interesse. Tra i numerosi incontri, pure quello con il collezionista e museologo svedese Ponthus Hultén (Stoccolma, 1924 - 2006), con l'artista greco residente a Roma Jannis Kounellis (Piraeus, 1936), con il pittore naif croato Ivan Rabuzin (Ključ, provincia di Novi Marof, 1921 - Varaždin, 2008) e con gli artisti americani Jim Dine (Cincinnati, 1935) e Claes Oldenburg (Stoccolma, 1929).

Nel capitolo sul "Golfo degli Angeli" Ghirardi sceglie le opere dellartista e pittore italiano Osvaldo Licini (Monte Vidon Corrado, 1894 - 1958) e quelle dello storico dell'arte Pietro Zampetti (Ancona, 1913 - Treviso, 2011), soffermandosi in particolare sulla mostra dedicata al pittore rinascimentale Carlo Crivelli (Venezia, 1430 - Ascoli Piceno, 1495) che Zampetti, in qualità di direttore dell'Accademia di Belle Arti, allestì nel 1961 al Palazzo Ducale di Venezia.

Nell'ottavo capitolo del libro Ghirardi ci offre un'analisi della Dalmazia vista quale territorio dai profondi contrasti, ma pure come "una terra dalle mille sorprese", osservata dagli occhi del summenzionato storico dellarte Giuseppe Maria Pilo. Ghirardi introduce nel testo anche i propri ricordi personali di questa interessante regione, che ricoprì un importante ruolo storico nel passato di questa parte d'Europa. Menziona, poi, tutta una serie di esperti che ne studiarono il ricco patrimonio storico-culturale, analizzato nella prospettiva della storia dell'arte (Gamulin, Fisković, Ivančević, Prijatelj, ecc.). Confronta infine la Dalmazia con l'Istria, sui cui affreschi aveva scritto alcuni decenni prima (G. Ghirardi, "Affreschi istriani del Medioevo", 1972). 
Trova posto nel volume anche il saggio sul pittore italiano Antonio Bellucci (Pieve di Soligo, 1654 - 1726), che ebbe come maestro Domenico Difnico di Sebenico. Egli lavorò pure a Venezia e diede un importantissimo contributo alla promozione della pittura veneziana in Austria ed in Inghilterra.

Alla fine del volume si leggono due testi critici scritti da quelli che furono collaboratori pluriennali di Ghirardi: i professori universitari emeriti dott. Miroslav Bertoša dell'Università Juraj Dobrila di Pola e dott. Paolo Leoncini dell'Università Cà Foscari di Venezia.

Nel proprio testo, scritto in lingua francese, Miroslav Bertoša tratta l'opera ghirardiana "Anime di confine" (Roma, 1999), ribadendo che si tratta di un autore dall'"irrefrenabile spirito creativo al quale l'ambiente professionale risulta chiaramente troppo stretto", e pertanto "amplia il proprio interesse di ricerca anche al contesto dell'esperienza letteraria, poetica, saggistica e pubblicistica. Assorto e profondamente immerso negli abissi della complessa realtà del passato, del presente e nel presagio di un futuro ancora più astruso, lo studioso veneziano professore d'arte e testimone del tempo, nella serie delle sue opere scritte, tutte concepite diversamente, distingue nettamente il drammatico problema dei confini della futura Europa e del mondo nel suo insieme". Inoltre, ribadisce Bertoša, "i testi di Ghirardi sono scritti con uno stile peculiare, stracolmi dellenorme sapere di un intellettuale appassionato lettore, ricchi di esperienza originale e corroborati da fedeli testimonianze della realtà, prevalentemente di frammenti della stessa".

L'articolo del professor Leoncini costituisce, a dire il vero, la versione scritta della sua lezione sull'opera di Giulio Ghirardi, tenuta in qualità di ospite verso la fine del mese di aprile 2013 all'Università Juraj Dobrila di Pola. Leoncini pone in luce soprattutto i "temi istriani" dell'immensa opera di Ghirardi, sottolineandone lo stile letterario ben curato, che nel racconto raggiunge "in profondità e in ampiezza il pensiero e il sentimento".

Lo scrittore, poeta, prosatore e saggista (come amava definire sé stesso) Giulio Ghirardi (Venezia, 6-III-1944 - 1-XI-2014) si è dedicato per circa un ventennio alla critica pittorica, pubblicando volumi ed articoli di ragguardevole spessore in varie riviste, per passare poi negli anni Novanta ad altri generi letterari. La sua opera ha attirato l'attenzione di moltissimi studiosi, letterati e critici. Ed è proprio nel libro del quale trattiamo in questa sede - e che costituisce pure la sua ultima opera stampata - che il prof. Giuseppe Maria Pilo lo descrive come saggista di enorme cultura e profondità umana, 
scrittore sempre imprevedibile, pensatore dotato di raro talento di ricerca, enorme conoscitore della gente, delle cose e degli eventi mitteleuropei. Al centro dell'interesse di Ghirardi c'è sempre stato l'Adriatico, Venezia soprattutto, e l'autore ha spesso trattato temi incentrati sullarte e sui costumi dei vari Paesi dell'area.

Giulio Ghirardi ha creato diligentemente per molti anni la sua enorme opera e per ben un trentennio ad affiancarlo nel suo lavoro vi sono state la moglie Elžbieta Waluk Ghirardi e la figlia MariaVittoria: ne sono nati preziosi ed interessanti lavori (libri, saggi, articoli-dibattiti, pensieri critici, ecc.) che, grazie allo stile transdisciplinare caratteristico dell'autore, toccano le sfere della storia dell'arte, della critica pittorica, dell'erudizione storica, del pensiero filosofico, letterario, ecc... Utile fonte di consultazione per vari studi incentrati su temi veneziani in senso lato, la sua opera, per la sua particolare importanza, rimarrà indelebilmente presente nelle sfere intellettuali italiane, ed in particolar modo veneziane, anche come fonte ispiratrice di ricerche future.

prof. dr. sc. Slaven Bertoša Università Juraj Dobrila di Pola Facoltà di Filosofia 